\section{Validade e reprodutibilidade de um questionário para medida de atividade física em adolescentes: uma adaptação do Self- Administered Physical Activity Checklist}

\section{Validity and reproducibility of $a$ physical activity questionnaire for adolescents: adapting the Self- Administered Physical Activity Checklist}

José Cazuza de Farias Júnior'

Adair da Silva Lopes"

Jorge Mota"'

Maria Paula Santos"I'

José Carlos Ribeiro"'

Pedro Curi Hallal'v

' Universidade Federal da Paraíba - CCS/DEF/UFPB e Grupo de Estudos e Pesquisas em Epidemiologia da Atividade Física - GEPEAF

" Universidade Federal de Santa Catarina - CDS/UFSC

II Universidade do Porto, Portugal e Centro de Investigação em Actividade Física, Saúde e Lazer - CIAFEL

Iv Universidade Federal de Pelotas - UFPel

\section{Resumo}

Objetivo: Analisar a reprodutibilidade "teste-reteste" e a validade de um questionário de atividade física em adolescentes (adaptado do Self-Administered Physical Activity Checklist). Métodos: A análise da reprodutibilidade "teste-reteste" foi realizada com 239 adolescentes (média de idade = 16,0 anos, $\mathrm{DP}=1,2 ; 56,4 \%$ do sexo feminino) e a análise da validade com 70 adolescentes (média de idade $=15,7$ anos, DP $=1,2 ; 55,7 \%$ do sexo feminino). Todos eram adolescentes do ensino médio no município de João Pessoa (PB), região Nordeste do Brasil, em 2009. A reprodutibilidade foi determinada por meio do procedimento de medidas repetidas - "teste-reteste", com uma semana de intervalo entre as réplicas de aplicação do questionário. Para avaliar a validade, comparou-se a medida de atividade física do questionário com a medida de quatro recordatórios de 24 horas de atividades físicas - método de referência. Resultados: A reprodutibilidade "teste-reteste" foi elevada (coeficiente de correlação intraclasse: CCI = 0,88; IC95\%: 0,84-0,91) e a concordância moderada para medida de atividade física em duas categorias (<300min/sem $v s$. $\geq 300 \mathrm{~min} / \mathrm{sem}$, índice kappa: $\mathrm{k}=0,52$ ). O coeficiente de correlação entre a medida de atividade física do questionário e a do recordatório de $24 \mathrm{~h}$ foi moderado (Spearman's rho $=0,62 ; \mathrm{p}<0001)$. A concordância entre estas duas medidas para o nível de atividade física em duas categorias foi moderada $(\mathrm{k}=0,59)$. Conclusões: O questionário apresentou níveis elevados de reprodutibilidade e moderados níveis de validade, podendo ser utilizado para mensurar o nível de atividade física em adolescentes.

Palavras-chave: Validade. Reprodutibilidade. Questionário. Atividade Física. Métodos. Adolescente. 


\section{Abstract}

Objectives: To evaluate test-retest reproducibility and the validity of a physical activity questionnaire for adolescents (adapted from Self-Administered Physical Activity Checklist). Methods: The test-retest reproducibility analysis was completed by 239 adolescents (average age 16 years, $\mathrm{SD}=1.2$; $56.4 \%$ female), and the validity analysis by 70 adolescents (average age 15.7 years, SD $=1.2 ; 55.7 \%$ female). All adolescents were high school students in João Pessoa, PB, in Northeastern Brazil, in 2009. The test-retest reproducibility was determined by repeated evaluations with one week interval between them. Validity of the physical activity questionnaire was assessed by comparing the results of the questionnaire with those of four 24-hour recalls (reference method). Results: The test-retest reproducibility was high (intraclass correlation coefficient: ICC $=0.88$; 95\%CI: $0.84-0.91$ ), and the agreement was moderate for physical activity classified in two groups (<300min/week vs. $\geq 300 \mathrm{~min} /$ week; kappa coefficient: $k=0.52$ ). Spearman correlation coefficient comparing the questionnaire data with those of four 24-hour recalls (reference method) was moderate (rho $=0.62 ; \mathrm{p}<0001)$. The agreement between these measures (questionnaire and 24-hours recalls) for physical activity classified in two groups was moderate ( $\mathrm{k}$ $=0.59$ ). Conclusions: The physical activity questionnaire showed high reproducibility, and moderate validity, and therefore, its use is recommended for assessing adolescents' physical activity level.

Keywords: Validity. Reliability. Questionnaire. Physical activity. Methods. Adolescent.

\section{Introdução}

Em estudos epidemiológicos com grande número de participantes, o nível de atividade física vem sendo mensurado essencialmente por meio de questionários ${ }^{1-4}$. No entanto, transcorridos quase 60 anos desde as primeiras publicações de estudos utilizando questionários de atividade física, a precisão e acurácia desses instrumentos ainda continuam sendo limitadas e questionadas ${ }^{5}$, principalmente em crianças e adolescentes $^{6}$.

Diversos questionários de atividade física têm sido testados em adolescentes ${ }^{2,3,7}$. Esses instrumentos têm demonstrado maior reprodutibilidade "teste-reteste" do que validade. Os níveis de reprodutibilidade "teste-reteste" têm variado de $0,20^{8}$ a $0,98^{9}$ e os de validade de $-0,13^{10}$ a $0,88^{11}$. Os questionários disponíveis na literatura mensuram de diferentes formas as atividades físicas em um ou mais domínios, por diferentes períodos de tempo, variando de um dia até um "histórico de atividades físicas na vida" ${ }^{2,3,7,12}$.

A maioria dos estudos ${ }^{2,3,7,12}$ que analisou a reprodutibilidade "teste-reteste" e validade dos questionários foi realizada em adolescentes de países desenvolvidos, em amostras por conveniência ou de voluntários, não envolveu adolescentes de diferentes faixas etárias, e a análise da validade foi efetuada por meio de coeficientes de correlação linear, os quais avaliam a magnitude da relação linear entre duas variáveis, mas não a concordância entre elas ${ }^{13}$.

Há poucos estudos publicados sobre a validade de questionários de atividade física testados em adolescentes brasileiros. Os que foram desenvolvidos focaram adolescentes das regiões Sul ${ }^{14-16}$ e Sudeste do país ${ }^{17}$, mas raramente da região Nordeste ${ }^{16}$. Um determinado questionário pode apresentar níveis satisfatórios de reprodutibilidade e validade em uma determinada população, mas necessariamente não terá o mesmo desempenho quando aplicado em sujeitos que apresentam características distintas daquelas apresentadas pela população de referência ${ }^{6}$. 
A aplicação de um questionário em sujeitos que apresentam características distintas daquelas a partir das quais o mesmo foi desenvolvido e avaliado pode resultar em viés "cultural" . Nesse sentido, torna-se necessário realizar uma adaptação cultural (estruturação, linguagem, atividades físicas habituais) e testar o instrumento antes da sua aplicação na população alvo do estudo. Em função das particularidades apresentadas pelas cidades da região Nordeste do Brasil (clima, hábitos de atividade física, denominação de algumas atividades físicas, características ambientais e socioculturais), este estudo foi desenvolvido com o objetivo de avaliar a reprodutibilidade e a validade de um questionário de atividade física em adolescentes.

\section{Métodos}

Em 2009 foi realizado um estudo transversal com adolescentes do ensino médio, de escolas públicas estaduais e privadas, no município de João Pessoa (PB), para avaliar a reprodutibilidade e a validade de um questionário de atividade física.

Para o cálculo do tamanho da amostra, considerou-se um coeficiente de correlação intraclasse (CCI) igual ou superior a $0,20^{2,3}$ para a reprodutibilidade, e um coeficiente de correlação igual ou superior a $0,40^{2,3}$ para a validade, um erro tipo I de $5 \%$ e erro tipo II de $20 \%$ e acréscimo de $30 \%$ para possíveis perdas/recusas, resultando em uma amostra de 195 adolescentes para a análise da reprodutibilidade e de 65 para a análise de validade.

A coleta de dados foi efetuada (de abril a maio de 2009) por equipe treinada, composta por seis estagiários do curso de Educação Física da Universidade Federal da Paraíba, supervisionados pelo pesquisador principal. Os estagiários receberam um manual com o protocolo do estudo, com o intuito de padronizar a coleta de dados, e passaram por um estudo piloto sob as mesmas condições do estudo principal.

O questionário foi respondido em sala de aula, durante o horário normal de aula, pelos próprios adolescentes, a partir de orientações prévias fornecidas por um estagiário, enquanto outro circulava em sala para ajudar a esclarecer as dúvidas por parte dos adolescentes.

Para caracterização da amostra estudada, os adolescentes responderam a questões sobre aspectos sociodemográficos (sexo, idade, escolaridade do pai e da mãe [fundamental incompleto, fundamental completo, médio incompleto, médio completo, ensino superior incompleto, superior completo]) e classe econômica (Associação Brasileira das Empresas de Pesquisa - ABEP, que agrupa as pessoas nas seguintes classes: A1 [maior nível], A2, B1, B2, C1, C2, D e E [menor nível] ${ }^{18}$.

O questionário de atividade física analisado no presente estudo é uma adaptação do Self-Administered Physical Activity Checklist $^{19}$. Uma adaptação deste questionário foi utilizada para mensurar o nível de atividade física em adolescentes do Sul do Brasil $^{20,21}$. Esses instrumentos também foram considerados na construção da versão que será analisada neste estudo (algumas atividades físicas).

Um estudo piloto (79 adolescentes de ambos os sexos, 14 a 19 anos de idade, escola pública e privada) foi realizado com o intuito de definir as atividades físicas que iriam compor o questionário e suas respectivas denominações (calibrar a lista de atividades segundo os hábitos de atividade física e as características culturais da população alvo). A versão final do questionário foi composta por uma lista com 24 atividades físicas de intensidade moderada a vigorosa $(\geq 3 \mathrm{METs})^{22}$, com possibilidade de o adolescente acrescentar mais duas (anexo). No preenchimento do questionário, os adolescentes informaram a frequência (dias/sem) e a duração (horas/min/dia) das atividades físicas praticadas na última semana.

Na determinação do nível de atividade física considerou-se o somatório do produto do tempo despendido em cada uma das atividades físicas pelas respectivas frequências de prática, seguindo o procedimento descrito em anexo. Foram considerados suficientemente ativos os adolescentes com 
prática de atividade física igual ou superior a 300min/sem e os demais como insuficientemente ativos ${ }^{23}$.

\section{Reprodutibilidade}

Para a análise da reprodutibilidade foram selecionados 336 escolares do ensino médio, de ambos os sexos. A amostra foi selecionada em dois estágios: seleção aleatória das escolas (4 escolas públicas e 2 privadas); seleção aleatória das turmas (18 turmas de ensino médio, uma turma por série de ensino em cada escola selecionada).

A reprodutibilidade foi estimada por meio do procedimento de medidas repetidas, com intervalo de uma semana entre as aplicações. Na primeira aplicação, os adolescentes receberam um envelope contendo duas cópias do instrumento, ambas com o mesmo código de barras. $\mathrm{O}$ adolescente retirou uma das cópias, lacrou o envelope com uma etiqueta, colocou o seu nome sobre a mesma e devolveu o envelope para o estagiário responsável pela aplicação. $\mathrm{Na}$ segunda aplicação, o adolescente recebeu o envelope com o seu nome, retirou o lacre e preencheu a réplica do questionário, seguindo as mesmas instruções.

\section{Validade}

Para a análise da validade, foram selecionados aleatoriamente 90 adolescentes de ambos os sexos ( 5 escolares por turma), dentre os que haviam respondido a primeira aplicação do questionário. Adotou-se como critério de referência a medida de atividade física obtida por meio de um recordatório-24h de atividades físicas ${ }^{24,25}$. Este instrumento demonstrou níveis elevados de reprodutibilidade e validade ${ }^{25,26} \mathrm{e}$ foi utilizado como critério de referência em outros estudos realizados com adolescentes ${ }^{2,3}$.

$\mathrm{O}$ instrumento foi aplicado individualmente, na forma de entrevista, durante quatro dias da semana, sendo dois dias do meio da semana e dois dias do final de semana. Para minimizar possíveis problemas de recordação, os adolescentes registraram as atividades físicas praticadas nas últimas 24 horas para os dias do meio de semana, e na segunda-feira responderam sobre as atividades físicas praticadas no final de semana (sábado e domingo).

Para proceder ao seu preenchimento, a cada intervalo de 15 minutos os adolescentes informaram a atividade física praticada durante 96 períodos, totalizando as $24 \mathrm{~h}$ do dia. As atividades referidas pelos adolescentes foram alocadas pelo entrevistador em nove grupos (1 [menor nível de intensidade] a 9 [maior nível de intensidade]), conforme os seus equivalentes energéticos ${ }^{22,25}$. Uma lista prévia de atividades físicas foi utilizada $^{22}$. Efetuou-se uma adaptação nas atividades sugeridas na versão original do recordatório- $24 \mathrm{~h}^{24}$ para torná-las mais próximas daquelas praticadas por adolescentes $^{25,26}$. Para fins de análise, foram considerados os dados dos adolescentes que preencheram os quatro recordatórios-24h.

Para determinar o nível de atividade física dos adolescentes, efetuou-se o somatório do tempo despendido nas atividades físicas de intensidade moderada a vigorosa $(\geq 3 \mathrm{METs})^{22}$, em média (ponderada), por semana. A classificação do nível de atividade física seguiu os mesmos critérios da medida de atividade física do questionário ${ }^{23}$.

\section{Análise dos dados}

A normalidade dos dados contínuos de atividade física foi avaliada por meio do teste de Kolmogorov-Smirnov. Os dados contínuos do nível de atividade física (réplicas de aplicação do questionário, recordatório-24h) não apresentaram distribuição normal. Para contornar o problema da normalidade, efetuou-se a transformação logarítmica dos dados.

O CCI foi utilizado para determinar a reprodutibilidade "teste-reteste" do questionário. As medidas de concordância entre as réplicas de aplicação para a medida de atividade física em duas categorias incluíram o percentual de concordância e o kappa ${ }^{27}$.

A validade foi determinada por meio do coeficiente de correlação de Spearman's 
( $r h o$ ). A medida de atividade física determinada a partir da segunda aplicação do questionário foi comparada contra a de quatro recordatórios de $24 \mathrm{~h}$ de atividades físicas.

O diagrama de dispersão de BlandAltman ${ }^{28}$ foi utilizado para verificar a concordância absoluta entre réplicas de aplicações do questionário e entre a medida de atividade física do questionário e aquela fornecida pelo método de referência. A concordância entre os dois métodos para a medida de atividade física em duas categorias foi avaliada por meio do percentual de concordância e do índice kappa.

Os dados foram digitados em duplicata no programa EpiData 3.1, com checagem automática de consistência e amplitude. A ferramenta validar dupla digitação deste programa foi utilizada para identificar erros de digitação. As análises estatísticas foram efetuadas no Stata 10.1, adotando-se um nível de significância de 5\%.

\section{Resultados}

Dos 336 adolescentes inicialmente selecionados para participar do estudo, 17 foram excluídos por terem $<14$ ou $>19$ anos de idade, 57 não estavam presentes na sala de aula na segunda aplicação do questionário, 9 não responderam as questões de atividade física na segunda aplicação do questionário, 14 se recusaram a participar e dois não foram autorizados pelos pais ou responsáveis. A análise da reprodutibilidade foi realizada com 239 adolescentes de 14 a 19 anos de idade (média de idade $=16,0$, DP $=1,2 ; 55,6 \%$ do sexo feminino) e a análise da validade com 70 adolescentes (média de idade $=15,6 ; \mathrm{DP}=1,2 ; 55,7 \%$ do sexo feminino) (Tabela 1). Na análise da validade, foram registradas 20 perdas (transferência, $\mathrm{n}=1$; desistência do ano escolar, $\mathrm{n}=7$; recusa em participar, $n=4$; não preencheram os quatro recordatórios, $\mathrm{n}=8$ ).

Não foram observadas diferenças significativas em relação ao nível de atividade física entre os adolescentes que responderam as duas aplicações do questionário e os que responderam apenas a primeira aplicação $(\mathrm{p}=0,20)$, e entre os que preencheram e os que não preencheram ou não preencheram os quatro recordatórios- $24 \mathrm{~h}(\mathrm{p}=0,09$ ).

Os valores médios do tempo despendido em atividades moderadas a vigorosas e a prevalência de adolescentes que atendiam as recomendações dos $300 \mathrm{~min} / \mathrm{sem}$ foram mais elevados na primeira aplicação do questionário (média = 519,7; IC95\%: 448,4 - 591,1; 52,7\%; IC95\%: 46,3 - 59,1) comparado à segunda (média $=401,6$; IC95\%: 340,6 - 462,7; 43,5\%; IC95\%: 37,2 - 49,8), mas sem diferenças significativas (interseção do IC95\%).

A Tabela 2 apresenta os resultados da análise da reprodutibilidade "teste-reteste". Todos os CCI foram superiores a 0,80 , sendo ligeiramente mais elevados nos adolescentes do sexo feminino (CCI = 0,88, IC95\%: 0,84 - 0,91 ) e nos mais jovens (14 - 15 anos, CCI = 0,88, IC9\%: 0,82-0,92). A concordância entre as réplicas de aplicação do questionário para o nível de atividade física em duas categorias foi moderada, kappa variando de 0,42 a 0,58 .

A Figura 1 apresenta o diagrama de dispersão de Bland-Altman para a concordância absoluta entre as duas aplicações do questionário. Verificou-se uma elevada concordância entre as réplicas de aplicação do questionário, sendo a diferença média entre a primeira e a segunda igual a 118,1 (IC95\%: 69,1 - 167,1), com limites de concordância variando de $871,1(+2 \mathrm{DP})$ a - 639,4 (- 2DP).

A comparação entre a medida de atividade física do questionário e do recordatório-24h de atividades físicas não demonstrou diferenças estatisticamente significativas, tanto nos dados contínuos (questionário: média $=464,5 ;$ DP = 551,9; IC95\%: 330,7 597,9 e recordatório-24h: média $=442,9$; $\mathrm{DP}=498,7 ;$ IC95\%: 324,0 - 561-9) quanto nos categóricos (questionário: 49,0\%; IC95\%: 36,8 - 59,2; recordatório-24h: 47,1\%; IC95\%: $35,1-59,2)$.

O questionário demonstrou níveis moderados de validade ( $\mathrm{rho}=0,62, \mathrm{p}<0,001$ ) ao ser comparado com o recordatório-24h (Tabela 3). Os coeficientes de correlação foram similares em ambos os sexos, porém 
Tabela 1 - Características da amostra, João Pessoa (PB), 2009.

Table 1 - Characteristics of the sample, João Pessoa (PB), 2009.

\begin{tabular}{|c|c|c|c|c|}
\hline \multirow[t]{2}{*}{ Variável } & \multicolumn{2}{|c|}{ Reprodutibilidade* } & \multicolumn{2}{|c|}{ Validade* } \\
\hline & $\mathrm{n}$ & $\%$ & $\mathrm{n}$ & $\%$ \\
\hline \multicolumn{5}{|l|}{ Sexo } \\
\hline Masculino & 106 & 44,6 & 31 & 44,3 \\
\hline Feminino & 133 & 56,4 & 39 & 55,7 \\
\hline \multicolumn{5}{|l|}{ Idade } \\
\hline 14-15 anos & 121 & 50,6 & 39 & 55,7 \\
\hline 16-19 anos & 118 & 49,4 & 31 & 44,3 \\
\hline \multicolumn{5}{|l|}{ Escola } \\
\hline Pública & 163 & 68,2 & 44 & 63,2 \\
\hline Privada & 76 & 31,8 & 26 & 36,8 \\
\hline \multicolumn{5}{|l|}{ Escolaridade do pai } \\
\hline Fundamental incompleto & 71 & 30,6 & 19 & 27,9 \\
\hline Médio incompleto & 47 & 20,3 & 18 & 26,5 \\
\hline Médio completo & 75 & 32,3 & 24 & 35,3 \\
\hline Superior completo & 39 & 16,8 & 7 & 10,3 \\
\hline \multicolumn{5}{|l|}{ Escolaridade da mãe } \\
\hline Fundamental incompleto & 60 & 25,2 & 21 & 30,4 \\
\hline Médio incompleto & 55 & 23,1 & 20 & 29,0 \\
\hline Médio completo & 81 & 34,0 & 22 & 31,8 \\
\hline Superior completo & 42 & 17,7 & 6 & 8,8 \\
\hline \multicolumn{5}{|l|}{ Classe econômica } \\
\hline A1/A2 (maior nível) & 15 & 6,7 & 1 & 1,6 \\
\hline $\mathrm{B} 1 / \mathrm{B} 2$ & 89 & 39,6 & 30 & 41,3 \\
\hline $\mathrm{C} 1 / \mathrm{C} 2$ & 101 & 44,9 & 26 & 47,6 \\
\hline D/E (menor nível) & 20 & 8,8 & 6 & 9,5 \\
\hline
\end{tabular}

${ }^{*}$ O número de itens sem resposta variou de $2(0,83 \%)$ a $14(5,9 \%)$ para a reprodutibilidade e de $1(1,43 \%)$ a $7(10,0 \%)$ para a validade.

*The number of unanswered items ranged from $2(0.83 \%)$ to 14 (5.9\%) for reproducibility and from 1 (1.43\%) to (10.0\%) for validity.

Tabela 2 - Medidas de reprodutibilidade "teste-reteste" do questionário, considerando a medida contínua de atividade física e em duas categorias, João Pessoa (PB), 2009.

Table 2 - Test-retest reproducibility of the questionnaire, considering the continuous measure of physical activity, and in two categories, João Pessoa (PB), 2009.

\begin{tabular}{lcccc}
\hline Variável & $\mathrm{CCl}$ & $\mathrm{IC} 95 \%$ & $\% \mathrm{C}$ & kappa \\
\hline $\begin{array}{l}\text { Todos } \\
\text { Sexo }\end{array}$ & 0,88 & $0,84-0,91$ & 75,7 & 0,52 \\
$\quad$ Masculino & 0,82 & $0,73-0,88$ & 71,7 & 0,43 \\
$\quad$ Feminino & 0,88 & $0,83-0,91$ & 78,9 & 0,54 \\
$\quad \begin{array}{l}\text { Idade } \\
\quad 14-15 \text { anos }\end{array}$ & 0,88 & $0,82-0,92$ & 72,7 & 0,42 \\
$\quad 16-19$ anos & 0,87 & $0,81-0,91$ & 78,8 & 0,58 \\
\hline
\end{tabular}

CCI: coeficiente de correlação intraclasse / ICC: intraclass correlation coefficient

IC95\%: intervalo de confiança a 95\% / 95\%Cl: confidence interval

\%C: Coeficiente de concordância / \%C: concordance coefficient 


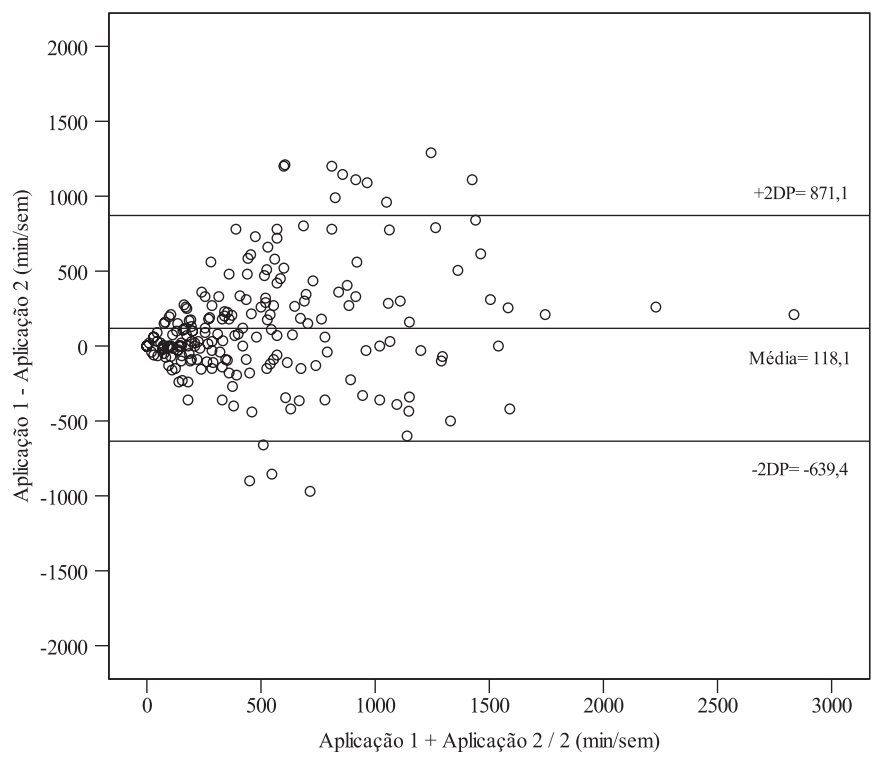

Figura 1 - Gráfico de Bland-Altman para verificar o grau de concordância entre as réplicas de aplicação - "teste-reteste" - do questionário de atividade física, João Pessoa (PB), 2009.

Figure 1 - Bland and Altman scatter plot comparing the score test and retest of the physical activity questionnaire, João Pessoa (PB), 2009.

ligeiramente superiores nos adolescentes mais velhos $(16-19$ anos, rho $=0,60 ; \mathrm{p}=$ $0,001)$ comparados aos mais jovens (14 - 15 anos, rho $=0,52 ; \mathrm{p}=0,001)$. A concordância entre os dois instrumentos para o nível de atividade física em duas categorias foi moderada $(k=0,59)$, sendo mais elevada nos adolescentes do sexo feminino $(\mathrm{k}=0,69)$ em comparação com seus pares do sexo masculino $(\mathrm{k}=0,41)$.

A Figura 2 apresenta o diagrama de Bland-Altman para a concordância entre a medida de atividade física do questionário e do recordatório. Verificou-se uma elevada concordância entre as medidas produzidas por esses dois instrumentos. A diferença média foi igual a 19,2 (IC95\%: -101,3 - 139,7), com limites de concordância que variaram (+ 2DP) 994,9 a (- 2DP) - 956,5. Percebe-se um aumento na discrepância entre as medidas dos dois instrumentos com aumento no nível de atividade física, sem a presença de erro sistemático (correlação entre a média das diferenças e a média das duas medidas: rho $=0,08 ; \mathrm{p}=0,24)$.

Tabela 3 - Medidas de validade do questionário de atividade física em adolescentes, João Pessoa (PB), 2009.

Table 3 - Validity of the physical activity questionnaire for adolescents, João Pessoa (PB), 2009.

\begin{tabular}{lcccc}
\hline Variável & Spearman's rho & Valor-p & $\% C$ & kappa \\
\hline Todos & 0,62 & $<0,001$ & 79,4 & 0,59 \\
Sexo & & & & \\
$\quad$ Masculino & 0,52 & 0,003 & 76,0 & 0,41 \\
$\quad$ Feminino & 0,51 & 0,001 & 86,6 & 0,69 \\
Idade & & & & \\
$\quad 14-15$ anos & 0,52 & 0,001 & 79,0 & 0,58 \\
$\quad 16-19$ anos & 0,60 & 0,001 & 80,0 & 0,61 \\
\hline
\end{tabular}

\%C: percentual de concordância / \%C: concordance percentage 


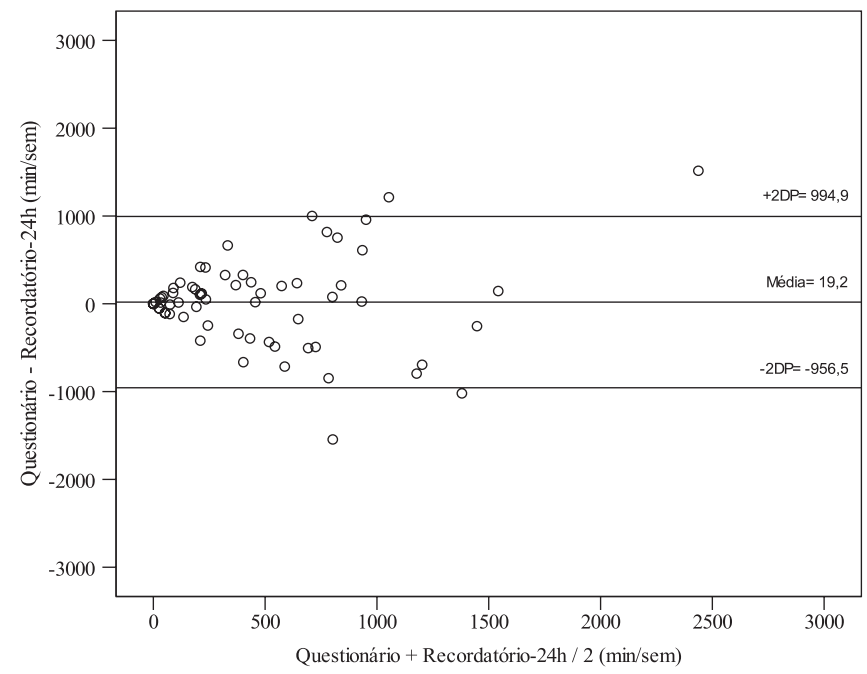

Figura 2 - Gráfico de Bland-Altman para verificar o grau de concordância entre a medida de atividade física do questionário e do recordatório-24h de atividades físicas ( $\mathrm{min} / \mathrm{sem})$, João Pessoa (PB), 2009.

Figure 2 - Bland and Altman scatter plot comparing the score test of the physical activity questionnaire and the 24-hour physical activity recall (min/week), João Pessoa (PB), 2009.

\section{Discussão}

O questionário de atividade física para adolescentes - QAFA apresentou níveis elevados de reprodutibilidade "teste-reteste", coeficientes de correlação moderados e boa concordância absoluta quando comparado à medida de atividade física derivada de um recordatório- $24 \mathrm{~h}$ de atividades físicas. Também houve uma concordância moderada entre os dois instrumentos para a medida de atividade física em duas categorias.

Um dos pontos fortes deste estudo foi ter analisado a reprodutibilidade e a validade do questionário em uma amostra aleatória e representativa da população adolescente do ensino médio de João Pessoa (PB). Normalmente, estes estudos têm sido desenvolvidos em amostras intencionais, por conveniência ou com a participação de voluntários ${ }^{2,3,7}$.

Outro ponto forte foi a realização de um estudo piloto para ajustar a lista e os nomes das atividades físicas que foram utilizadas no questionário. Destaca-se também o fato de o estudo ter utilizado uma amostra de tamanho adequado, com poder $(\beta)$ variando de $83 \%$ a $99 \%$ para as medidas de validade $(\alpha=5 \%)$ e superior a $95 \%$ para a reprodutibilidade $(\alpha<1 \%)$.

Uma das limitações deste estudo foi ter utilizado uma medida subjetiva de atividade física como critério de referência - "padrão-ouro". Embora os recordatórios-24h tenham demonstrado boa concordância com as medidas objetivas de atividade física em adolescentes ${ }^{3}$, podem ser acometidos por problemas de recordação e de estimação das informações que estão sendo mensuradas $^{3}$. Esses instrumentos, assim como as demais medidas subjetivas de atividade física, também podem ser influenciados por respostas que são socialmente aceitas ou esperadas por determinados grupos sociais ${ }^{3,6}$.

Acredita-se que a adoção de algumas medidas pode ter contribuído para minimizar e/ou evitar o impacto de alguns desses fatores. Por exemplo, o recordatório foi aplicado sob a forma de entrevista e os adolescentes recordaram as atividades físicas praticadas no dia anterior (maior precisão das informações recordadas). $\mathrm{O}$ recordatório utilizado neste estudo demonstrou níveis elevados de concordância com algumas medidas objetivas de atividade física (acelerômetros, monitores de frequência cardíaca e água duplamente marcada-DLW) $)^{25,26}$. 
Outro aspecto a ser considerado é que problemas de precisão no método de referência não devem produzir superestimação da validade do método ou instrumento que está sendo testado, mas sim uma subestimação das suas medidas de validade ${ }^{29}$. Apesar de as medidas objetivas serem recomendadas para servir de "padrão ouro" para validar medidas subjetivas (questionários, diários, recordatórios, entrevistas) ${ }^{3}$, ainda não está estabelecido se há verdadeiramente um "padrão ouro" adequado, contra o qual os questionários de atividade física possam ser avaliados/testados ${ }^{1}$. Diferentes métodos têm sido utilizados na validação dos questionários, produzindo medidas de validade de diferentes magnitudes ${ }^{2,3,7}$.

O questionário de atividade física analisado neste estudo apresentou níveis elevados de reprodutibilidade "teste-reteste", com todos os CCI superiores a 0,80. Além disso, verificou-se uma concordância absoluta elevada entre as suas réplicas de aplicação ("teste-reteste"). O valor da diferença média foi relativamente baixo $(118,1$; IC9\%: 69,1 - 167,1), não há excesso de valores discordantes de elevada magnitude, e quase todas as diferenças individuais entre os pares de aplicação do questionário estão dentro dos limites recomendados ${ }^{28}$.

Tem-se observado uma grande variação nos coeficientes de reprodutibilidade "teste-reteste" dos questionários de atividade física em adolescentes, de $0,20^{8}$ a $0,98^{9}$. Os resultados deste estudo foram superiores aos descritos em alguns estudos prévios com adolescentes ${ }^{14,15,19}$, mas inferiores a outros $^{11,30}$.

Em estudo realizado com adolescentes de Pelotas, RS, região Sul do Brasil, Bastos et al. ${ }^{20}$ encontram níveis de reprodutibilidade inferiores aos que foram encontrados neste estudo, para uma versão similar do questionário analisado neste estudo $(\mathrm{CCI}=0,62$; $\mathrm{p}<0,001)$. Essas discrepâncias podem ser atribuídas às diferenças nas idades dos adolescentes que participaram dos dois estudos (Pelotas, RS: 10 a 19 anos; João Pessoa, PB: 14 a 19 anos). As características psicológicas, cognitivas e de padrões de atividade física dos adolescentes mais jovens tornam o processo de recordação das atividades mais complexo e menos preciso, resultando em menores níveis de reprodutibilidade ${ }^{6}$.

Outros questionários testados em adolescentes brasileiros demonstraram níveis de reprodutibilidade inferiores aos que foram encontrados neste estudo (Questionário de avaliação da atividade física para adolescentes $^{17}$, ICC $=0,61$; Questionário internacional de atividade física ${ }^{14}$, rho = 0,59; Questionário Baecke $^{15}$, rho $=0,73$ ), exceto para o módulo de atividade física do Questionário Saúde na Boa $(\mathrm{ICC}=0,93)^{16}$.

A consistência entre as duas aplicações do questionário para medida de atividade física em duas categorias foi moderada (kappa variando de 0,42 a 0,58). Apesar de o índice kappa ser influenciado pela prevalência do desfecho e por "viés de medida", esse fenômeno não foi observado no presente estudo. Os valores do kappa ajustado, denominado de kappa PABAK ${ }^{31}$, foram similares aos do kappa "normal".

Como neste estudo os adolescentes recordaram as atividades físicas praticadas em diferentes períodos (semanas distintas) nas duas aplicações, mudanças ocorridas no nível de atividade física nesse período podem ter contribuído para a subestimação das medidas de reprodutibilidade. Especula-se que caso tivesse sido utilizado o mesmo período de recordação nas duas aplicações, os níveis de reprodutibilidade teriam sido ainda mais elevados.

O intervalo entre as réplicas de aplicação do questionário pode influenciar os níveis de reprodutibilidade. Utilizar intervalos prolongados entre as réplicas de aplicação do instrumento pode favorecer mudanças nas atividades físicas praticadas, resultando em subestimação da reprodutibilidade. Caso o intervalo seja muito curto, pode-se ter o "efeito memória", resultando em superestimação da reprodutibilidade do instrumento. Recomenda-se que o intervalo entre as aplicações do questionário seja curto, de um a três dias, não superior a uma semana, com sobreposição no período de recordação das atividades físicas ${ }^{2,32}$. 
A elevada consistência entre as réplicas de aplicação do questionário observada no questionário analisado neste estudo sugere que ele poder ser aplicado por diferentes pesquisadores de forma padronizada, e que os adolescentes não apresentaram dificuldades para compreensão das questões: informar a frequência e a duração das atividades físicas praticadas na última semana.

Em relação à validade, verificaram-se coeficientes de correlação moderados entre a medida do questionário e a do recordatório-24h de atividades físicas (rho $=0,62 ; \mathrm{p}<0,001)$. Apesar da grande quantidade de estudos sobre validade de questionários de atividade física em adolescentes ${ }^{2,3,7}$, a comparabilidade entre eles é muito limitada, devido às diferenças nas características da amostra, mas sobretudo devido às particularidades de cada questionário. Há diferenças acentuadas na forma como os questionários operacionalizam e expressam a medida de atividade física em adolescentes ${ }^{2,3}$. Em geral, a maioria dos coeficientes de correlação dos questionários avaliados em adolescentes tem sido inferior a $0,50^{2,3}$. Poucos questionários alcançaram coeficientes de correlação acima de $0,50^{2,3,7}$.

Bastos et al..$^{20}$ testaram uma versão similar do questionário avaliado neste estudo em adolescentes de Pelotas, RS. Os autores encontraram um coeficiente de correlação igual a 0,69 ao comparar o questionário com outro mais longo e de rho $=0,34$ para a medida do pedômetro. Os coeficientes de validade encontrados neste estudo também foram superiores aos que haviam sido observados em estudos com adolescentes brasileiros, que avaliaram outros questionários (Questionário de avaliação da atividade física para adolescentes ${ }^{17}$, ICC $=0,15$ a 0,23; Questionário internacional de atividade física ${ }^{14}$, rho = 0,26; Questionário Baecke $^{15}, \mathrm{k}=0,44$; módulo de atividade física do Questionário Saúde na Boa ${ }^{16}$, rho = 0,41).

Tendo em vista que os coeficientes de correlação de Pearson e Spearman indicam apenas a magnitude da relação linear entre duas variáveis ${ }^{28}$, mas não a concordância entre elas, efetuou-se análise de concordância entre a medida do questionário e a do recordatório-24h. Para tanto, utilizou-se o método de Bland-Altman ${ }^{28}$. O valor da diferença média foi relativamente baixo (19,2; IC9\%: -101,3 - 137,3), e não há excesso de valores discordantes de elevada magnitude entre as duas medidas de atividade física.

Embora tenha sido observado um aumento na discrepância entre as medidas dos dois instrumentos com aumento do nível de atividade física, o coeficiente de correlação entre a média das diferenças entre as duas medidas de atividade física (recordatório-24h vs. questionário) e a média das mesmas não foi significante ( $r h o=0,08 ; p=0,24)$. $O$ aumento na discrepância entre as medidas de atividade física dos dois instrumentos era um resultado já esperado, por se tratar de uma medida de concordância absoluta. Além disso, os adolescentes mais ativos fisicamente tendem a superestimar a participação em atividades físicas, sobretudo nas atividades vigorosas ${ }^{26}$.

Apesar de a medida de atividade física durante quatro dias ser recomendada quando se utiliza instrumento que mensura a atividade física em único dia ${ }^{1}$, não se pode descartar a hipótese de que aplicar o recordatório-24h durante quatro dias pode não caracterizar o nível de atividade física atual dos adolescentes. Desse modo, as medidas de validade deste estudo poderiam ter sido mais elevadas caso sete recordatórios-24h fossem preenchidos.

Ainda não há um valor de referência em relação aos coeficientes de correlação que defina o que representa um instrumento com níveis satisfatórios de validade. A seguinte conclusão foi apresentada por diversos autores quando os instrumentos apresentam correlações entre 0,30 e 0,50 : "o instrumento apresentou níveis satisfatórios de validade e pode ser utilizado para mensurar a atividade física"14,16,19,30. Podese assumir que o questionário analisado neste estudo alcançou níveis satisfatórios de validade.

Dentre as principais vantagens do QAFA, destaca-se a sua capacidade em mensurar 
tipo, intensidade, frequência e duração das atividades físicas praticadas e a possibilidade de realizar estimativas de atividade física segundo as recomendações atuais para adolescentes ${ }^{23}$, bem como a facilidade de aplicação e de preenchimento por parte dos adolescentes.

Os níveis reprodutibilidade do QAFA foram elevados. O coeficiente de correlação para validade foi moderado, mas superior ao que tem sido encontrado na maioria dos estudos, principalmente com adolescentes brasileiros. A concordância entre a medida do questionário e do recordatório-24h foi de moderada a elevada para a medida de atividade física em duas categorias e elevada para a medida contínua. O questionário analisado neste estudo apresentou níveis satisfatórios de reprodutibilidade e validade, podendo ser utilizado para mensurar a atividade física em adolescentes.

\section{Aspectos éticos}

O estudo foi aprovado pelo Comitê de Ética em Pesquisa com Seres Humanos do Centro de Ciências da Saúde da Universidade Federal da Paraíba (Protocolo $\mathrm{n}^{\circ}$ 0062). Todos os adolescentes menores de 18 anos de idade receberam autorização dos pais ou responsáveis para participar do estudo.

\section{Referências}

1. Dollman J, Okely AD, Hardy L, Timperio A, Salmon J, Hills AP. A hitchhiker's guide to assessing young people's physical activity: deciding what method to use. J Sci Med Sport 2009; 12: 518-25.

2. Sallis JF, Saelens BE. Assessment of physical activity by self-report: status, limitations, and future directions. Res Q Exerc Sport 2000; 71(2): 1-14.

3. Sirard J, Pate RR. Physical activity assessment in children and adolescents. Sports Medicine 2001; 31(6): 439-54.

4. Dumith SC. Physical activity in Brazil: a systematic review. Cad Saúde Pública 2009; 25(3): S415-26.

5. Shephard R. Limits to the measurement of habitual physical activity by questionnaires. Br J Sports Med 2004; 37: 197-206.

6. Baranowski T. Validity and reliability of self-report measures of physical activity: an information-processing perspective. Res Q Exerc Sport 1988; 59(4): 14-27.

7. Adamo KB, Prince SA, Tricco AC, Connor-Gorber S, Tremblay M. A comparison of indirect versus direct measures for assessing physical activity in the pediatric population: a systematic review. Int J Pediatr Obes 2009; $42-27$.

8. Brown TD, Holland BV. Test-retest reliability of the selfassessed physical activity checklist. Percept Mot Skills 2004; 99: 1099-102.

9. Allor KM, Pivarnik JM. Stability and convergent validity of three physical activity assessments. Med Sci Sports Exerc 2001; 33(4): 671-76.

10. Crocker PRE, Holowachuk DR, Kowalski KC. Feasibility of using the tritrac motion sensor over a 7-day trial with older children. Ped Exerc Sci 2001; 13: 70-81.
11. Weston AT, Petosa R, R PR. Validation of an instrument for measurement of physical activity in youth. Med Sci Sports Exerc 1997; 29(1): 138-43.

12. Corder K, Ekelund U, Steele MR, Wareham NJ, Brage O. Assessment of physical activity in youth. J Appl Physiol 2008; 105: 977-87.

13. Bland JM, Altman DG. Comparing methods of measurement: why plotting difference against standard method is misleading. Lancet 1995; 346(21): 1085-7.

14. Guedes DP, Lopes CC, Guedes JERP. Reprodutibilidade e validade do questionário internacional de atividade física em adolescentes. Rev Bras Med Esporte 2005; 11(2): 151-8.

15. Guedes DP, Lopes CC, Guedes JERP, Stanganelli LC. Reprodutilibidade e validade do questionário Baecke para avaliação da atividade física habitual em adolescentes. Rev Port Cien Desp 2006; 6(3): 265-74.

16. Nahas MVN, Barros MVG, Florindo AA, Farias Junior JC, Hallal PC, Konrad L, et al. Reprodutibilidade e validade do questionário saúde na boa para avaliar atividade física e hábitos alimentares em escolares do ensino médio. RBAFS 2007; 12(3): 12-20.

17. Florindo AA, Romero A, Peres SV, Silva MV, Slater B. Desenvolvimento e validação de um questionário de avaliação da atividade física para adolescentes. Rev Saude Publica 2006; 40(5): 802-09.

18. ABEP (Associação Brasileira das Empresas de Pesquisa). Critério de Classificação Econômica Brasil. Disponível em http://www.abep.org.br. (Acessado em 26 de novembro de 2009). 
19. Sallis JF, Strikmiller PK, Harsha DW, Feldman HA, Ehlinger S, Stone EJ. Validation of interviewer and selfadministered physical activity checklists for fifth grade students. Med Sci Sports Exerc 1996; 28(7): 840-51.

20. Bastos JP, Araújo CLP, Hallal PC. Prevalence of insufficient physical activity and associated factors in brazilian adolescents. J Phys Act Health 2008; 7: 777-94.

21. Farias Júnior JC, Nahas MV, Barros MVG, Loch MR, Oliveira ESA, De Bem MFL et al. Comportamentos de risco à saúde em adolescentes no Sul do Brasil: prevalência e fatores associados. Rev Panam Salud Publica 2009; 25(4) 1-11.

22. Joanne SH, Mcmurray RG, Baggettchristopher D, Pennell M, Pearce P, Bangdiwala SI. Energy costs of physical activities in children and adolescents. Sports Exerc Med Sci 2005; 37(2): 326-36.

23. Biddle S, Sallis JF, Cavill NA. Young and active? Young people and health enhancing physical activity. Evidence and implication. London: Health Education Authority; 1998.

24. Bouchard C, Tremblay A, Leblanc C, Lortie G, Sauard R, Theriault G. A method to assess energy expenditure in children and adults. Am J Clin Nutr 1983; 37: 461-7.

25. Bratteby L-E, Sandhagen B, Fan H, Samuelson G. A 7-day activity diary for assessment of daily energy expenditure validated by the doubly labelled water method in adolescents. Eur J Clin Nutr 1997; 51: 585-91.
26. Ekelund U, Yngvel A, Sjostrom M. Total daily energy expenditure physical activity in adolescents different methods. Scand J Med Sci Sports 1999; 9: 257-64.

27. Landis JR, Koch GG. The measurement of observer agreement for categorical data. Biometrics 1997; 33(1): 159-74.

28. Bland JM, Altmand G. Statistical methods for assessing agreement between two methods of clinical measurement. Lancet 1986; 1: 307-10.

29. Monteiro CA, Florindo AA, Claro RM, Moura EC. Validade de indicadores de atividade física e sedentarismo obtidos por inquérito telefônico. Rev Saúde Pública 2008; 42(4): 575-81.

30. Ridley K, Olds TS, Hill A. The multimedia activity recall for children and adolescents (MARCA): development and evaluation. Int J Behav Nutr Phys Act 2006; 3(10): doi:10.1186/479-5868-3-10.

31. Byrt T, Bishop J, Carlin JB. Bias, Prevalence and Kappa. J Clin Epidemiol 1993; 46(5): 423-9.

32. Patterson P. Reliability, validity, and methodological response to the assessment of physical activity via selfreport. Res Q Exerc Sport 2000; 71(2): 15-20.

Recebido em: 10/09/10

Versão final apresentada em: 24/12/10 Aprovado em: 11/04/11 


\section{Questionário de Atividade Física para Adolescentes - QAFA}

Para cada uma das atividades físicas listadas abaixo, você deverá responder quantos dias por semana e quanto tempo por dia, em média, você praticou na SEMANA PASSADA. Caso tenha praticado alguma atividade física que não esteja listada abaixo, escreva o(s) nome(s) da(s) atividade(s) no espaço reservado no final da lista (linhas em branco).

\begin{tabular}{|c|c|c|}
\hline \multirow{2}{*}{ Atividades físicas } & Quantos dias? & Quanto tempo cada dia? \\
\hline & 0 a 7 dias & Tempo (horas:minutos) \\
\hline Futebol (campo, de rua, society) & & _horas___minutos \\
\hline Futsal & & minutos \\
\hline Handebol & & _horas___minutos \\
\hline Basquete & & minutos \\
\hline Andar de patins, skate & & horas___ minutos \\
\hline Atletismo & & horas___ minutos \\
\hline Natação & & _horas___ minutos \\
\hline Ginástica olímpica, rítmica & & minutos \\
\hline Judô, karatê, capoeira, outras lutas & & _horas___ minutos \\
\hline Jazz, balê, dança moderna, outros tipos de dança & & minutos \\
\hline Correr, trotar (jogging) & & _horas___ minutos \\
\hline Andar de bicicleta & & horas___ minutos \\
\hline Caminhar como exercício físico & & _horas___ minutos \\
\hline $\begin{array}{l}\text { Caminhar como meio de transporte (ir à escola, trabalho, casa } \\
\text { de um amigo (a)). [Considerar o tempo de ida e volta] }\end{array}$ & & horas___ minutos \\
\hline Voleibol & & horas___ minutos \\
\hline Vôlei de praia ou de areia & & horas___ minutos \\
\hline Queimado, baleado, pular cordas & & _horas___ minutos \\
\hline Surfe, bodyboard & & minutos \\
\hline Musculação & & _horas___minutos \\
\hline Exercícios abdominais, flexões de braços, pernas & & horas___ minutos \\
\hline Tênis de campo (quadra) & & _horas___minutos \\
\hline Passear com o cachorro & & horas___ minutos \\
\hline Ginástica de academia, ginástica aeróbica & & minutos \\
\hline Futebol de praia (beach soccer) & & _horas ___ minutos \\
\hline \multirow[t]{3}{*}{ Outras atividades físicas que não estão na lista acima: } & & _horas___minutos \\
\hline & & _minutos \\
\hline & & horas___ minutos \\
\hline
\end{tabular}

Cálculo do nível de atividade física - NAF

NAF:

$-\min / \mathrm{sem} / \mathrm{AFMV}=\Sigma\left[\mathrm{F}_{\mathrm{i}} \times \mathrm{D}_{\mathrm{j}}\right]$

$-\min / \mathrm{dia} / \mathrm{AFMV}=\Sigma\left[F_{\mathrm{i}} \times D_{\mathrm{j}}\right] / 7$

\section{Onde:}

AFVM: atividades físicas moderadas a vigorosas

$\Sigma$ : somatório do produto da frequência (dias/sem) pela duração ( $\mathrm{min} / \mathrm{dia})$ da atividade física

$F_{i:}$ frequência da i-ésima atividade física

$D_{j}$ duração ( $\mathrm{min} /$ dia) da j-ésima atividade física 